\title{
Linking the chiral and deconfinement phase transitions
}

\author{
Yoshitaka Hatta ${ }^{1,2,3}$ and Kenji Fukushima ${ }^{4, *}$ \\ ${ }^{1}$ Department of Physics, Kyoto University, Kyoto 606-8502, Japan \\ ${ }^{2}$ The Institute of Physical and Chemical Research (RIKEN) Wako, Saitama 351-0198, Japan \\ ${ }^{3}$ High Energy Theory, Department of Physics, Brookhaven National Laboratory, Upton, NY 11973, U.S.A. \\ ${ }^{4}$ Department of Physics, University of Tokyo, 7-3-1 Hongo, Bunkyo-ku, Tokyo 113-0033, Japan
}

(Dated: October 16, 2018)

\begin{abstract}
We show that the electric glueball becomes critical at the end-point of the deconfinement phase transition in finite temperature QCD. Based on this observation and existing lattice data, we argue that the chiral phase transition at a zero quark mass and the deconfinement phase transition at an infinite quark mass are continuously connected by the glueball-sigma mixing.
\end{abstract}

PACS numbers: $25.75 . \mathrm{Nq}, 12.38 . \mathrm{Mh}$

Confinement and chiral symmetry breaking are the fundamental properties of quantum chromodynamics (QCD) in the nonperturbative regime. It has been often speculated how the two phenomena are related to each other. There are several arguments that the confined vacuum necessarily breaks chiral symmetry [1, 2], which suggest that the energy scale of chiral symmetry breaking is higher than that of confinement. The separation of these scales, i.e., $\sim 4 \pi f_{\pi}\left(f_{\pi}\right.$ is the pion decay constant) for chiral symmetry breaking and $\sim \Lambda_{\mathrm{QCD}}$ for confinement, has been successfully exploited to reproduce the low energy hadronic properties 3].

In view of such experiences at zero temperature, it is quite surprising that the lattice data at finite temperature suggest that chiral symmetry restoration and the deconfinement phase transition occur at the same temperature [4, 5, 6, 7, 8, 9, 10]. In this Brief Report we argue how this could be realized.

Fig. 1 is the schematic phase diagram of QCD in the $(T, m)$ plane. ( $T$ is the temperature and $m$ is the current quark mass.) The deconfinement phase transition has a definite meaning only at an infinite quark mass. In $\mathrm{SU}(3)$ pure gluodynamics the transition is known to be first order 11]. The expectation value of the Polyakov loop [12, 13],

$$
L(\boldsymbol{x})=\operatorname{tr} \mathcal{P} \exp \left(-\mathrm{i} \int_{0}^{1 / T} A_{0}(\boldsymbol{x}, \tau) \mathrm{d} \tau\right),
$$

where $\mathcal{P}$ is the path ordering operator, serves as an order parameter and jumps at the deconfinement transition temperature. The first order phase transition persists at a finite but very large quark mass (solid curve in Fig. 1) and eventually terminates at a second order phase transition point $\mathrm{D}$ where $\langle L(\boldsymbol{x})\rangle$ becomes continuous. The universality class of this phase transition is that of the three-dimensional Ising model. The critical quark mass at $\mathrm{D}$ is estimated to be $\sim \mathrm{O}(1) \mathrm{GeV}$ in the two-flavor lattice simulations [14, 15].

\footnotetext{
*Present address: Center for Theoretical Physics, Massachusetts Institute of Technology, Cambridge, MA 02139
}

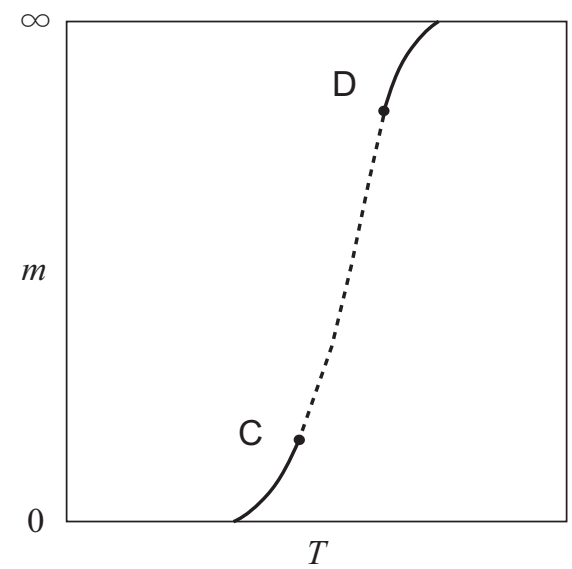

FIG. 1: The phase diagram in the $(T, m)$ plane

In contrast, chiral symmetry is a true symmetry only at zero quark mass. The order parameter of the chiral phase transition is the quark condensate $\langle\bar{q} q\rangle$. For three flavors, the phase transition is of first order [16]. With finite but small quark masses (which we take to be equal for all flavors), the first order transition persists and eventually terminates at a second order phase transition point C. The jump in the chiral condensate disappears and the sigma meson screening state $\sigma \sim \bar{q} q$ becomes gapless at this point [17, 18]. The location of $C$ and $D$ depends on the number of flavors and their mass ratios. [For example, $C$ lies on the $m=0$ axis for two flavors [16].] The following argument does not depend on the details of the flavor sector, however.

Between the points $C$ and $D$, there is no true phase transition. There are just two crossover phase transitions starting from $C$ and $D$. The location of the crossover transition (the pseudo-critical temperature) is determined from the peak position of the susceptibility of each order parameter. The lattice data suggest that the two pseudo-critical temperatures coincide for all values of the quark mass, which means that two crossover curves are smoothly connected at some intermediate point (dotted curve in Fig. 1). It is as if the deconfinement phase tran- 
sition in the pure gluonic theory is 'guided' towards the chiral phase transition traveling over an infinite range of the quark mass.

The nature of this 'coincidence' has been discussed in 19, 20, 21, 22], where the authors attributed the (almost) singular behavior of the Polyakov loop to the coupling of the Polyakov loop and the chiral condensate. It turns out that their observation can be incorporated in a very simple and intuitive picture which enables us to grasp what is going on near the finite temperature QCD phase transition for all values of the quark mass.

Since the center symmetry is explicitly broken by dynamical quarks [23], the Polyakov loop has a nonzero expectation value at $D$. Thus the phase transition at $D$ would be characterized by the infinite correlation length (or the vanishing screening mass) of the shifted order parameter

$$
L^{\prime}(\boldsymbol{x}) \equiv L(\boldsymbol{x})-\langle L\rangle,
$$

where $\langle L\rangle$ is the expectation value of $L$ at D. However, there is an apparent puzzle. What is the physical meaning of the 'mass' of the $L^{\prime}$ field? A naive guess is the string tension as in the case of the pure $\mathrm{SU}(2)$ theory. This is wrong because the string eventually breaks at large distances in the presence of dynamical quarks no matter how heavy they are, whereas the second order phase transition requires an infinite correlation length. We will show that the massless mode at $D$ is actually the scalar glueball screening state.

For this purpose, first we work in the pure $\mathrm{SU}(2)$ gauge theory. In this case the deconfinement phase transition is second order in the universality class of the three dimensional Ising model. The string tension $K$ goes to zero smoothly as the critical temperature is approached

$$
K \sim|t|^{\nu}, \quad(t<0)
$$

where $t$ is the reduced temperature and the universal exponent is $\nu \simeq 0.63[25]$.

One can realize the relevance of glueballs by considering the specific heat $C_{\mathrm{v}}$. In the theory of critical phenomena, the singular part of the specific heat is represented by the integrated correlator of the order parameter squared [26],

$$
C_{\mathrm{v}} \sim \int \mathrm{d}^{d} x\left\langle L^{2}(\boldsymbol{x}) L^{2}(\mathbf{0})\right\rangle_{\mathrm{c}} \sim|t|^{-\alpha},
$$

where $\alpha \simeq 0.11$ and $d=3$ is the spatial dimension. The subscript c denotes the connected part. (Note that $L(\boldsymbol{x})$ is real for $\mathrm{SU}(2)$ and $\left\langle L^{2}(\boldsymbol{x})\right\rangle$ stays nonzero at any temperature.) The scaling dimension of the order parameter squared is $d-1 / \nu[26]$. Thus the explicit structure of the divergence is

$$
\int \mathrm{d}^{d} x \frac{\mathrm{e}^{-m_{\mathrm{G}}|\boldsymbol{x}|}}{|\boldsymbol{x}|^{2\left(d-\frac{1}{\nu}\right)}} \sim|t|^{-\alpha},
$$

where we have assumed that the connected part of the correlator in (4) exponentially decays as $\sim \mathrm{e}^{-m_{\mathrm{G}}|\boldsymbol{x}|}$ at large distances. Matching the powers of $|t|$ and using Josephson's law $\alpha=2-d \nu$ (hyperscaling), we obtain,

$$
m_{\mathrm{G}} \sim|t|^{\nu} .
$$

Hence the operator $L^{2}$ couples to a state with zero screening mass at the phase transition. By using the $\mathrm{SU}(2)$ identity

$$
L^{2}(\boldsymbol{x})=1+L_{\mathrm{A}}(\boldsymbol{x}),
$$

where $L_{\mathrm{A}}$ is the Polyakov loop in the adjoint representation (i.e., the trace in Eq. (1) is taken in the adjoint representation), or just by noticing that $L^{2}$ is invariant under the center transformation, $L \rightarrow-L$, we see that the massless state is the glueball 27]. [More precisely, the electric glueball screening state. See below.]

It should be noted that Eq. (6) does not mean that the (electric) glueball is another order parameter field. Rather, it is a trivial consequence of the scaling property at general second order phase transitions; the only relevant scale is the correlation length $\xi \sim|t|^{-\nu}$. [The word 'massless' may be somewhat misleading since we normally associate it with the order parameter field, namely, the Polyakov loop.]

One might suspect that the vanishing of the glueball screening mass would contradict with the presence of the trace anomaly. In the simple phenomenological Lagrangian of glueballs at zero and low temperatures [29, 30], the glueballs acquire their mass entirely from the trace anomaly, or the gluon condensate. However, the exact lattice sum rule for the glueball mass 31. shows that only $\frac{1}{4}$ of the glueball mass comes from the expectation value of the trace anomaly in the glueball state measured relative to the vacuum. Moreover, the scalar glueball mass at zero temperature splits into two branches at finite temperature. The glueballs which become massless at the phase transition are the electric glueballs (those containing $A_{0}$, or time-like links), while the screening mass of the magnetic glueball (composed of $A_{i}$, or space-like links) remains finite. This crucial difference has to be taken into account in phenomenological models such as [32]. As long as the specific heat diverges in the usual manner at the $\mathrm{SU}(2)$ deconfinement phase transition 33], we believe that the vanishing of the electric glueball screening mass and the non-vanishing of the trace anomaly are compatible. A significant decrease of the electric glueball mass near the $\mathrm{SU}(2)$ deconfinement transition has been observed on the lattice [35].

Let us go back to the end-point D of SU(3) QCD with dynamical quarks. We can think of an effective GinzburgLandau theory near $\mathrm{D}$ in terms of the new order parameter $L^{\prime}$ defined in Eq.(2). However, due to our identification

$$
G(\boldsymbol{x}) \sim|L(\boldsymbol{x})|^{2}=\left|L^{\prime}(\boldsymbol{x})+\langle L\rangle\right|^{2},
$$

the glueball field $G(\boldsymbol{x})$ contains a linear term in $L^{\prime}$. Hence the screening mass of the Polyakov loop which vanishes at $D$ is equal to the screening mass of the glueball, and 
we may as well construct a Ginzburg-Landau theory of $G$ around D. [Note that in contrast to the $\mathrm{SU}(2)$ case, the electric glueball is the order parameter field in the case of $\mathrm{SU}(3)$.] The crucial point is that, in the presence of dynamical quarks, the electric glueballs must mix with the $\bar{q} q$ states. In fact, the correct massless field associated with the second order phase transition at $D$ is a linear combination of the pure glueball interpolating field $G$ and the sigma meson interpolating field $\sigma$ with large gluonic content,

$$
\phi=G \cos \theta+\sigma \sin \theta, \quad \sin \theta \ll 1 .
$$

The other linear combination with large mesonic content,

$$
\phi^{\prime}=-G \sin \theta+\sigma \cos \theta,
$$

is massive, hence decouples at $\mathrm{D}$.

The mixing Eq. (9) inevitably occurs if the first order phase transition line makes an angle with the quark mass axis at $\mathrm{D}$, and there is no symmetry to keep them parallel. In the same way, at $C$ the critical sigma screening state slightly mixes with the glueball screening state. The simultaneous enhancement of the chiral susceptibility

$$
\chi_{\mathrm{ch}} \sim \int \mathrm{d}^{d} x\langle\bar{q} q(\boldsymbol{x}) \bar{q} q(\mathbf{0})\rangle,
$$

and the Polyakov loop susceptibility along each crossover phase transition line is a trivial consequence of the mixing 21, 22].

Now we address a deeper question: The lattice data suggest that there is only one crossover phase transition line in the $(T, m)$ plane, not two. With the above line of argument we are led to the following scenario which naturally explains this longstanding puzzle. Let us decrease the quark mass $m$ and follow the crossover phase transition line starting from $D$. The screening mass of the $\phi$ field first increases from zero with a certain critical exponent. At the same time, the mixing angle $\theta$ gradually increases. If the mixing angle stops growing and stays small, the $\phi^{\prime}$ field, which is then always sigma-like, serves as the order parameter at C. In this case, the two phase transitions would have nothing to do with each other and there would be two peaks in both the chiral and Polyakov loop susceptibilities. [Remember that the coincidence occurs irrespective of whether or not the two crossover lines meet.] However, the observed single peak means that the $\phi$ field describes the field with the smallest screening mass (or the longest correlation length) in the system for all values of the quark mass and the mixing angle gradually changes from $\theta \sim 0$ to $\theta \sim \pi / 2$. Namely, the $\phi\left(\phi^{\prime}\right)$ field is first glueball-like (sigma-like) for large values of the quark mass and it becomes sigma-like (glueball-like) as the quark mass gets smaller. In order for this to be realized, a level repulsion between the $\phi$ state and the $\phi^{\prime}$ state must occur at an intermediate value of the quark mass. The fate of the $\phi$ field is predominantly sigma-like and is nothing but the order parameter of the genuine QCD phase transition, the chiral phase transition. This gives a simple and intuitive explanation of why there seems to be only one phase transition in finite temperature QCD lattice simulations.

An immediate consequence of the mixing Eq. (9) is that the electric glueball (or the Polyakov loop) and the sigma meson screening masses coincide at the common pseudo-critical temperature of the crossover phase transition. [Note that in principle, the mixing between the sigma and the glueball could occur in the entire phase diagram (Fig. 1). However, we expect that in most regions the mixing is too small to be observed on the lattice. For example, above the chiral phase transition temperature, at small quark masses the sigma meson and the pions fall in a multiplet due to (approximate) chiral symmetry. The glueball screening mass is indeed different from the sigma screening mass in this region [36]. Also, at low temperatures the glueball will primarily mix with nearby scalar quarkonium states. The point is that near the crossover phase transition, the sigma meson and the glueball conspire to create the lightest particle which drives the phase transition. At large distances, no other states can couple to this mode. Thus the glueball-sigma mixing and the resulting degeneracy in their screening mass should be observable in this region (but not necessarily in other regions).] Detailed lattice measurements of the correlators (or the off-diagonal correlators) of electric glueballs, Polyakov loops and sigma mesons around the region between $C$ and $D$ would give us the information of the lowest and the second lowest screening masses as well as the quark-mass dependence of the mixing angle. Compilation of such measurements would confirm or exclude our scenario.

It is intriguing to consider the phase diagram for different values of $N_{\mathrm{c}}$, the number of colors. As $N_{\mathrm{c}}$ increases, it is likely that the first order deconfinement phase transition strengthens [37] and the point D moves downward. Presuming that our scenario also holds for general $N_{\mathrm{c}} \geq 3$, we expect that the point $\mathrm{D}$ reaches the point $\mathrm{C}$ at some value of $N_{\mathrm{c}}=N_{\mathrm{c}}^{*}$ and the high and low temperature phases are separated by a single first order phase transition line for $N_{\mathrm{c}} \geq N_{\mathrm{c}}^{*}$. This is consistent with the early observations [38]. From our viewpoint, $N_{\mathrm{c}}=2$ is special. The Ginzburg-Landau theory of $G$ does not make sense at $\mathrm{D}$ which now lies on the $m=\infty$ axis. Also the nature of the chiral phase transition is peculiar for $N_{\mathrm{c}}=2$ due to the Pauli-Gürsey symmetry [39]. It is thus interesting to pursue a similar link (glueballsigma-diquark mixing) between the two phase transitions in $N_{\mathrm{c}}=2 \mathrm{QCD}$ at finite temperature and baryon density.

In conclusion, based on the existing lattice data, we have proposed a novel scenario of the finite temperature QCD phase transition. The chiral phase transition at zero quark mass and the deconfinement phase transition at infinite quark mass are continuously connected by the glueball-sigma mixing which is a well-known phenomenon in the zero temperature scalar meson spectrum. In other words, the two phase transitions are just different limits of a single (crossover) phase transition driven by a sin- 
gle field. Phenomenological consequences of this scenario remain to be explored.

\section{Acknowledgements:}

We thank M. Creutz, L. McLerran, P. Petreczky, R. D. Pisarski, K. Splittorff and M. A. Stephanov for their interest in this work and helpful comments. Y. H. thanks RIKEN BNL Reserch Center. K. F. is supported by the Japan Society for the Promotion of Science for Young Scientists.
[1] A. Casher, Phys. Lett. B 83, 395 (1979).

[2] G. 't Hooft, in Recent Developments in Gauge Theories, Eds. G. 't Hooft et al., (Plenum, New York, 1980).

[3] A. Manohar and H. Georgi, Nucl. Phys. B 234, 189 (1984).

[4] J. B. Kogut et al., Phys. Rev. Lett. 50, 393 (1983).

[5] M. Fukugita and A. Ukawa, Phys. Rev. Lett. 57, 503 (1986).

[6] F. Karsch and E. Laermann, Phys. Rev. D 50, 6954 (1994)

[7] S. Aoki et al., Phys. Rev. D 57, 3910 (1998).

[8] F. Karsch, E. Laermann and A. Peikert, Nucl. Phys. B 605, 579 (2001).

[9] C. Gattringer, P. E. Rakow, A. Schafer and W. Soldner, Phys. Rev. D 66, 054502 (2002).

[10] C. R. Allton et al., Phys. Rev. D 66, 074507 (2002).

[11] L. G. Yaffe and B. Svetitsky, Phys. Rev. D 26, 963 (1982).

[12] A. M. Polyakov, Phys. Lett. B 72, 477 (1978).

[13] L. Susskind, Phys. Rev. D 20, 2610 (1979).

[14] P. Hasenfratz, F. Karsch and I. O. Stamatescu, Phys. Lett. B 133, 221 (1983).

[15] N. Attig, B. Petersson, M. Wolff and R. V. Gavai, Z. Phys. C 40, 471 (1988).

[16] R. D. Pisarski and F. Wilczek, Phys. Rev. D 29, 338 (1984).

[17] S. Gavin, A. Gocksch and R. D. Pisarski, Phys. Rev. D 49, 3079 (1994)

[18] F. Karsch, E. Laermann and C. Schmidt, Phys. Lett. B 520, 41 (2001).

[19] A. Gocksch and M. Ogilvie, Phys. Rev. D 31, 877 (1985).

[20] C. X. Chen and C. DeTar, Phys. Rev. D 35, 3963 (1987).

[21] H. Satz, Nucl. Phys. A 642, 130c (1998); S. Digal, E. Laermann and H. Satz, Eur. Phys.8 J. C 18, 583 (2001).

[22] K. Fukushima, Phys. Lett. B 553, 38 (2003); Phys. Rev.
D 68, 045004 (2003).

[23] For an attempt to define an order parameter in the presence of dynamical quarks, see [24].

[24] K. Fukushima, Annals Phys. 304, 72 (2003).

[25] J. Engels et al., Nucl. Phys. B 280, 577 (1987).

[26] J. Zinn-Justin, Quantum Field Theory and Critical Phenomena, 3rd edition (Oxford University Press, 1996).

[27] The state created by the operator $L_{\mathrm{A}}$ is often referred as a 'gluelump' in distinction from a 'glueball' [28]. However, we expect that the difference vanishes near the critical point. Note that in practical lattice simulations the specific heat is measured from the integrated correlator of plaquettes which excite glueballs.

[28] C. Michael, Nucl. Phys. B 259, 58 (1985).

[29] J. Schechter, Phys. Rev. D 21, 3393 (1980).

[30] P. J. Ellis, J. I. Kapusta and H. B. Tang, Phys. Lett. B 443, 63 (1998).

[31] H. J. Rothe, Phys. Lett. B 364, 227 (1995).

[32] A. Mocsy, F. Sannino and K. Tuominen, hep-ph/0306069

[33] Due to the very small critical exponent, $\alpha \sim 0.11$, the correct singularity in the specific heat has not been confirmed on the lattice so far. See, however, [34].

[34] J. Engels, F. Karsch and K. Redlich, Nucl. Phys. B 435, 295 (1995)

[35] S. Datta and S. Gupta, Nucl. Phys. B 534, 392 (1998).

[36] R. V. Gavai and S. Gupta, Phys. Rev. Lett. 85, 2068 (2000)

[37] B. Lucini, M. Teper and U. Wenger, Phys. Lett. B 545 , 197 (2002); hep-lat/0307017

[38] F. Neri and A. Gocksch, Phys. Rev. D 28, 3147 (1983).

[39] See, Y. Nishida, K. Fukushima and T. Hatsuda, hep-ph/0306066 and references therein. 